\title{
Original
}

\section{Rapid Discrimination between Methicillin-Sensitive and Methicillin-Resistant Staphylococcus aureus Using MALDI-TOF Mass Spectrometry}

\author{
KAZUYUKI SOGAWA ${ }^{1 *}$, MASAHARU WATANABE ${ }^{2}$, TAKAYUKI ISHIGE ${ }^{2,3}$, \\ SYUNSUKE SEGAWA ${ }^{2,3}$, AKIKO MIYABE ${ }^{2}$, SYOTA MURATA ${ }^{2}$, TOMOKO SAITO ${ }^{2}$, \\ AKIHIRO SANDA ${ }^{1}$, KATSUNORI FURUHATA ${ }^{4}$, AND FUMIO NOMURA ${ }^{5}$ \\ 'Department of Biochemistry, School of Life and Environmental Science, Azabu University, \\ 1-17-71 Fuchinobe, Chuo-ku, Sagamihara, Kanagawa 252-5201, Japan \\ 2 Department of Clinical Laboratory, Chiba University Hospital, \\ 1-8-1 Inohana, Chuo-ku, Chiba, Chiba 260-8670, Japan \\ ${ }^{3}$ Department of Molecular Diagnosis, Graduate School of Medicine, Chiba University, \\ 1-8-1 Inohana, Chuo-ku, Chiba, Chiba 260-8670, Japan \\ ${ }^{4}$ Department of Microbiology, School of Life and Environmental Science, Azabu University, \\ 1-17-71 Fuchinobe, Chuo-ku, Sagamihara-shi, Kanagawa 252-5201, Japan \\ ${ }^{5}$ Divisions of Clinical Mass Spectrometry and Clinical Genetics, Chiba University Hospital, \\ 1-8-1 Inohana, Chuo-ku, Chiba-shi, Chiba 260-8670, Japan \\ Received 11 May, 2015/Accepted 1 March, 2017
}

\begin{abstract}
Methicillin-resistant Staphylococcus aureus (MRSA) is one of the major pathogens responsible for nosocomial infections. The presence of MRSA in a hospital is detrimental to patients and to hospital management. Thus, rapid identification of MRSA is needed. Here, we report on a prospective method to rapidly discriminate of MSSA from MRSA using matrix-assisted laser desorption ionization-time of flight mass spectrometry (MALDI-TOF MS) and support vector machine (SVM) analysis in 160 clinical isolates of $S$. aureus. The predictive model was tested using $100 \mathrm{~S}$. aureus isolates (50 MSSA and 50 MRSA). The identification rates were $90.0 \%$ for MSSA and $\mathbf{8 7 . 5} \%$ for MRSA in a 10-fold cross-validation SVM. In blind test sets, $60 \mathrm{~S}$. aureus isolates (30 MSSA and 30 MRSA) were correctly classified, with identification rates of $93.3 \%$ for MSSA and $86.7 \%$ for MRSA. The method proposed in this study using the predictive model enables detection of one colony in 5 minutes, and thus is useful at clinical sites at which rapid discrimination of MRSA from MSSA is required.
\end{abstract}

Key words : Rapid discrimination / MSSA / MRSA / MALDI-TOF MS / MALDI BioTyper software.

\section{INTRODUCTION}

Matrix-assisted laser desorption/ionization time-offlight mass spectrometry (MALDI-TOF MS) is increasingly used in clinical microbial diagnostics for species identification of pathogens (Nomura., 2015). Bacteria can be rapidly identified by comparing MALDI-TOF MS fingerprints to a database of reference spectra, and this

*Corresponding author. Tel·Fax: +81-42-769-1924, E-mail : sogawa (a)azabu-u.ac.jp technique has been used to identify microorganisms such as Gram-negative rods (e.g., Escherichia coli and other members of the Enterobacteriaceae family) (Camara et al., 2007), Gram-positive cocci (e.g., Staphylococcus aureus and Streptococcus) (EdwardsJones et al., 2000), and some Gram-positive rods (e.g., Bacillus cereus) (Ryzhov et al., 2000). MALDI-TOF MS for identification of bacterial strains isolated from clinical samples has been examined in detail (Seng et al., 2009). In our laboratory, we prospectively assessed the performance of MALDI-TOF MS for identification of 
bacterial strains that are routinely isolated from clinical samples. The identification rates at the species and genus levels were 91.7\% (429/468) and 97.0\% (454/ 468), respectively (Sogawa et al., 2011), and both rates were improved by adding clinical isolate data obtained in our laboratory to that in the original commercial database (Sogawa et al., 2012).

Methicillin-resistant Staphylococcus aureus (MRSA) is one of the major pathogens responsible for nosocomial infection. In Japan, MRSA was isolated from 59.7\% of cases diagnosed with respiratory tract infections involving S. aureus in 2007 (Niki et al., 2009). Bacteremia due to MRSA in compromised patients has markedly increased morbidity and mortality compared to that caused by methicillin-susceptible Staphylococcus aureus (MSSA) infection (Weinstein et al., 1997; Wisplinghoff et al., 2004). Treatment of MRSA-linked bacteremia is also associated with higher hospital costs and longer hospitalization, compared to MSSA-linked bacteremia (Ben-David et al., 2009). Therefore, the presence of MRSA is detrimental to patients and to hospital management, and early detection and breaking of the infection route are important for preventing infection and reducing the prevalence of MRSA in hospitals. However, S. aureus is mostly detected in a cultured colony by examinations such as a coagulase test, and confirmation of MRSA requires a further 24 hours (Lowy., 2003). In the current work, we performed a prospective study of a method to discriminate of MSSA from MRSA using MALDI-TOF MS, with the goal of the rapid identification of MRSA.

\section{MATERIALS AND METHODS}

\section{Clinical samples}

A total of $160 \mathrm{~S}$. aureus isolates were tested using MALDI-TOF MS over a 9-month period from October 13, 2013 to January 14, 2015 in Chiba University Hospital. The isolates were retrieved from clinical samples and identified in our laboratory at the species level in parallel with use of conventional methods. During the first six months, $100 \mathrm{~S}$. aureus isolates (50 MSSA and 50 MRSA) (set 1) were subjected to MALDI-TOF MS analysis. The MALDI system results of pattern-matching are expressed as scores from 0 to 3 , with a score $<1.7$ indicating unreliable identification; $>1.7$ indicating genus identification, and $>2.0$ indicating species identification. The isolates were successfully identified by the MALDI system with a score $>2.0$ and their spectral data were incorporated into the original database. Using the new algorithm (which we refer to as the MRSA algorithm), $60 \mathrm{~S}$. aureus isolates (30 MSSA and 30 MRSA) (set 2) obtained in the next 3 months and $5 S$. aureus isolates (4 MSSA and one MRSA) (set 3) transferred Gifu University School of Medicine Pathogenic Bacterial
Resorce Stock Center (GMGC) were tested. Each clinical specimen was plated on an appropriate agar plate (Chocolate Agar, Trypticase Soy Agar II with 5\% sheep blood; Nippon Becton Dickinson Co., Tokyo, Japan), depending on the sample and in accordance with our laboratory procedures.

\section{Conventional identification}

The isolates underwent Gram staining and their catalase and oxidase activities were determined. They were identified using phenotypic tests, including the MicroScan WalkAway system (Siemens Healthcare Diagnostics, Deerfield, IL, USA), and oxacillin and cefoxitin MICs were determined. The presence of penicillin-binding protein $2 \mathrm{a}$ ( $\mathrm{PBP} 2 \mathrm{a})$ and mecA genes was suspected when the oxacillin and cefoxitin breakpoints were $\geq 4$ and $\geq 8$, respectively. The presence of PBP2a was confirmed using a MRSA-LA test (Denka Seiken Co., Niigata, Japan) and that of mecA was confirmed by PCR.

\section{MALDI-TOF MS}

To identify the isolates, MALDI-TOF MS was performed on a AutoFlex ${ }^{\circledR}$ || TOF/TOF mass spectrometer (Bruker Daltonics $\mathrm{GmbH}$, Leipzig, Germany) with Flexcontrol ${ }^{\mathrm{TM}}$ software 3.0 (Bruker Daltonics) for automatic acquisition of mass spectra in the linear positive mode within a range of 2 to $20 \mathrm{kDa}$. The mass spectrometer was periodically calibrated using a Bruker Daltonics bacterial test standard (Escherichia coli extracts including additional RNase A and myoglobin proteins). Calibration masses were as follows: RL36, 4364.3 Da; RS22, 5095.8 Da; RL34, 5380.4 Da; RLmeth, 6254.4 Da; RL32, 6315.2 Da; RL29, 7273.5 Da; RS19, 10229.1 Da; RNase A, 13682.2 Da; myoglobin, 16952.5 Da. Automated analysis of the raw spectral data was performed using MALDI BioTyper automation 2.0 software (Bruker Daltonics) based on standard pattern matching using a library of 3,746 spectra (database updated on June 9, 2010) and the default settings.

A single colony was directly deposited on a MTP BigAnchorChip 384 TF target plate (Bruker Daltonics). The preparation was overlaid with $1 \mu \mathrm{l}$ of $a-\mathrm{CHCA}$ matrix solution (a saturated solution of $a$-cyano4-hydroxycinnamic acid in 50\% acetonitrile/2.5\% trifluoroacetic acid). The colony was then air dried at room temperature to allow co-crystallization with the experimental sample.

The process from MALDI-TOF MS measurement to identification was performed automatically without user intervention. Briefly, the software generated a list of peaks up to 100. The threshold for peak acceptance was a signal-to-noise ratio of 10. After alignment, peaks with a mass-to-charge ratio difference $<250$ ppm were considered to be identical. The generated peak lists 
were used for matches against the reference library using the integrated pattern matching algorithm in the software. Results of pattern-matching are expressed as scores from 0 to 3 , with a score $<1.7$ indicating unreliable identification; $>1.7$ indicating genus identification, and $>2.0$ indicating species identification (Sogawa et al., 2011; 2012).

\section{Support vector machine (SVM) classification}

SVM is a new machine learning approach originally proposed and developed by Vapnik (Liu et al., 2004). SVM applications are being pursued in areas from face recognition to genomics (Liu et al., 2006). SVM has been used to identify candidate molecular markers predicting chemotherapy resistance in non-small cell lung cancer (Han et al., 2010) and to analyze serum peptidome profiles in breast cancers (Fan et al., 2012). This approach could be a powerful tool for analysis of complex data from MALDI-TOF MS. We constructed a non-linear SVM classifier to discriminate between MRSA and MSSA. Visual evaluation of MALDI-TOF spectra was performed using FlexAnalysis 3.0 (Bruker Daltonics). For construction of the algorithm, raw spectra were processed using the SVM approach in MALDI BioTools 3.0.

\section{Protein Identification}

One colony was suspended in $300 \mu \mathrm{L}$ of distilled RNasefree water. Absolute ethanol $(900 \mu \mathrm{L})$ was added and mixed carefully, and then the sample was centrifuged $(14,000 \times g$ for $2 \mathrm{~min})$. The supernatant was discarded and the pellet was air dried. Formic acid (70\%; $25 \mu \mathrm{L}$ ) and acetonitrile $(25 \mu \mathrm{L})$ were added to the pellet and mixed thoroughly, and then the sample was centrifuged again $(14,000 \times g$ for $2 \mathrm{~min})$. Ten volumes of ice cold acetone were added to the supernatant $(40 \mu \mathrm{L})$. Peptides/proteins were allowed to precipitate at $-20^{\circ} \mathrm{C}$ for $2 \mathrm{~h}$ and then obtained by centrifugation $(13000 \times \mathrm{g}$ for $10 \mathrm{~min}$ at $4^{\circ} \mathrm{C}$ ). After decanting of acetone, the peptides/proteins were air dried. The dried pellets were resuspended in buffer ( $0.1 \%$ trifluoroacetic acid in water, $\mathrm{vol} / \mathrm{vol}$ ) and further separated by reversed-phase HPLC in an automated HPLC system (Shiseido Nanospace SI-2, Shiseido Fine Chemicals, Tokyo, Japan).

The concentrated flow-through sample $(75 \mu \mathrm{L})$ was directly loaded onto a Capcell Core C18 column (Shiseido). Reversed-phase separations for each flowthrough fraction were performed using a multisegment elution gradient with eluent A $(0.1 \%$ trifluoroacetic acid in water, vol $/ \mathrm{vol})$ and eluent $\mathrm{B}(0.08 \%$ trifluoroacetic acid in $90 \%$ acetonitrile, vol/vol). The gradient elution program consisted of three steps with increasing concentrations of eluent B ( $5 \%$ B for 5 min, $5 \%$ to $95 \%$ $\mathrm{B}$ for $23 \mathrm{~min}$, and 95\% B for $11 \mathrm{~min}$ ) followed by $5 \% \mathrm{~B}$ for $21 \mathrm{~min}$ for re-equilibration of the column at a flow rate of $0.40 \mathrm{~mL} / \mathrm{min}$, with a total run time of $60 \mathrm{~min}$. Based on the chromatogram recorded by measuring the absorbance of the eluate at $280 \mathrm{~nm}$, fractions eluted at retention times between 19.1 and 39.1 min were collected in $400.2 \mathrm{~mL}$ aliquots at a fraction size setting of $0.5 \mathrm{~min}$. Fractions including objective peaks were confirmed by MALDI-TOF MS. N-terminal amino acid sequence analysis was performed using a Procise 494 CLC protein sequencing system (Applied Biosystems, Foster City, CA, USA).

\section{Immunoprecipitation}

One colony was suspended in $300 \mu \mathrm{L}$ of distilled RNasefree water. Absolute ethanol $(900 \mu \mathrm{L})$ was added and mixed carefully, and then the sample was centrifuged (14,000 $\times g$ for $2 \mathrm{~min})$. Supernatant was discarded and the pellet was air dried. Formic acid (70\%; $25 \mu \mathrm{L})$ and acetonitrile $(25 \mu \mathrm{L})$ were added to the pellet and mixed thoroughly, and then the sample was centrifuged again $(14,000 \times g$ for $2 \mathrm{~min})$. Ten $\mathrm{mg}$ of antibody-fixed affinity beads (Shiseido) was bound with $10 \mu \mathrm{g}$ of MRSA PBP2a monoclonal antibody (Abnova Corp., Taipei, Taiwan). Subsequently, $40 \mu \mathrm{L}$ of supernatant was mixed with $100 \mu \mathrm{g}$ of beads in $500 \mu \mathrm{L}$ of $20 \mathrm{mM}$ Tris-buffered saline at $4^{\circ} \mathrm{C}$ for $1 \mathrm{~h}$. After centrifugation $(3000 \times g$ for 1 min at $4^{\circ} \mathrm{C}$ ), the pellets were eluted with $100 \mu \mathrm{L}$ of 100 $\mathrm{mM}$ glycine- $\mathrm{HCl}$. The tube was placed in a Magnetic Separator and the supernatant was collected for further pretreatment with C18 Magnetic Beads (Bruker Daltonics).

\section{RESULTS}

\section{Development of the discrimination algorithm}

In the first set of $100 \mathrm{~S}$. aureus isolates (50 MSSA and 50 MRSA), all were correctly identified at the species level with a score $>2.0$. The concordance of identification at the species level was 100.0\% (100/100 isolates) compared with the conventional method. Visual evaluation of MALDI-TOF spectra was performed using FlexAnalysis 3.0 (Fig.1). After noise filtering and peak clustering, 128 peaks were detected in the MSSA and MRSA groups. All mass peaks were fed into the SVM and the accuracy of each combination for distinguishing MSSA from MRSA was analyzed. This model comprised 6 peaks with $\mathrm{m} / \mathrm{z}$ of $1888.1,1935.9,2760.3,2867.9$, 3044.2, and 4641.3. The average intensities for the 6 peaks are shown in Table 1. For other peaks, there was no significant difference $(p<0.05)$ between MRSA and MSSA. The predictive model was tested using $100 \mathrm{~S}$. aureus isolates (50 MSSA and 50 MRSA). The identification rates were $90.0 \%$ for MSSA and $87.5 \%$ for MRSA following the 10-fold cross-validation SVM. 

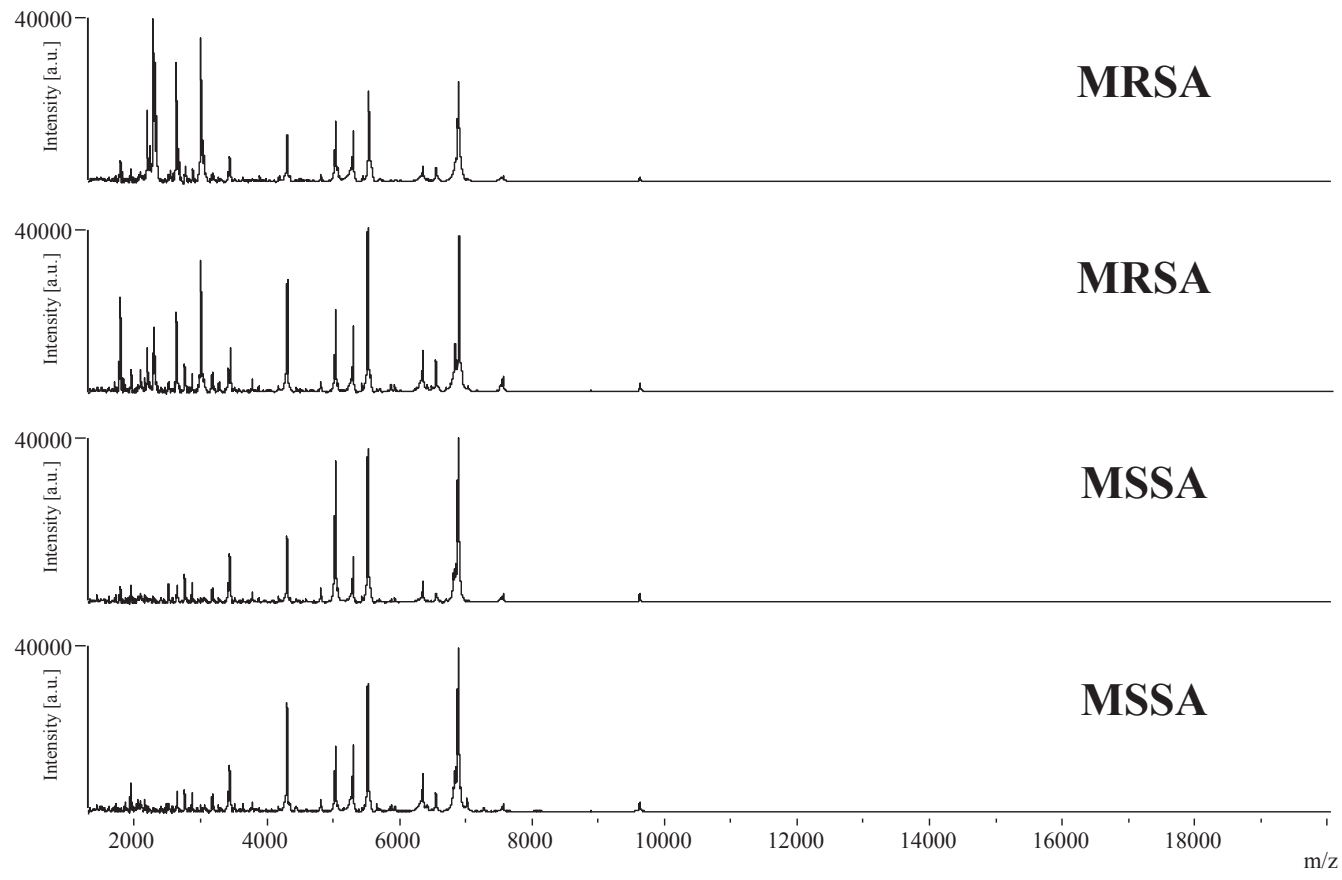

FIG. 1. Representative spectra of $S$. aureus methicillin-susceptible isolates and methicillin-resistant isolates.

TABLE 1. List of peaks and weights used in the discrimination algorithm

\begin{tabular}{cccc}
\hline Peak No. & $m / z$ & $\begin{array}{c}\text { MRSA } \\
\text { average intensity } \\
\text { (A. U.) }\end{array}$ & $\begin{array}{c}\text { MSSA } \\
\text { average intensity } \\
\text { (A. U.) }\end{array}$ \\
\hline 1 & 1888.1 & 430.3 & 90.2 \\
2 & 1935.9 & 880.8 & 662.2 \\
3 & 2760.3 & 663.6 & 1230.1 \\
4 & 2867.9 & 1490.9 & 601.3 \\
5 & 3044.2 & 20061.4 & 102.7 \\
6 & 4641.3 & 260.0 & 32.5 \\
\hline
\end{tabular}

\section{Evaluation of the discrimination algorithm}

In the second set of $60 \mathrm{~S}$. aureus isolates (30 MSSA and $30 \mathrm{MRSA}$ ), all were again correctly identified at the species level with a score $>2.0$. The concordance of identification at the species level was 100.0\% (100/100 isolates) compared with the conventional method. In the blind test sets, $60 \mathrm{~S}$. aureus isolates (30 MSSA and 30 MRSA) were correctly classified, with identification rates of $93.3 \%$ for MSSA and $86.7 \%$ for MRSA (Table 2).

In the third set of 5 S. aureus isolates (4 MSSA and one MRSA), all were again correctly identified at the species level with a score $>2.0$. The concordance of identification at the species level was 100.0\% (100/100 isolates) compared with the conventional method. Five
TABLE 2. Comparison of $60 \mathrm{~S}$. aureus isolates (30 MSSA and $30 \mathrm{MRSA}$ ) identified by the conventional method and the MALDI-TOF MS method

\begin{tabular}{|c|c|c|c|c|}
\cline { 3 - 5 } \multicolumn{2}{c|}{} & \multicolumn{3}{c|}{ Conventional method } \\
\cline { 3 - 5 } \multicolumn{2}{c|}{} & MRSA & MSSA & Total \\
\hline \multirow{3}{*}{$\begin{array}{c}\text { MALDI-TOF MS } \\
\text { method }\end{array}$} & MRSA & 26 & 2 & 28 \\
\cline { 2 - 5 } & MSSA & 4 & 28 & 32 \\
\cline { 2 - 5 } & Total & 30 & 30 & \\
\hline
\end{tabular}

S. aureus isolates (4 MSSA and one MRSA) were correctly classified, with identification rates of $100.0 \%$ for MSSA and $100.0 \%$ for MRSA.

\section{Identification of the 3043 Da peptide}

Partial purification of the peptide corresponding to the $3043 \mathrm{~m} / \mathrm{z}$ peak and $\mathrm{N}$-terminal amino acid sequencing revealed that the 3043 Da peptide was a fragment of PBP2a (Fig.2).

The intensity is very low in other 5 peaks, it could not be identified.

\section{Immunoprecipitation}

The PBP2a level in the supernatant was minimal when the MRSA isolate was incubated with antibody-fixed affinity beads coated with MRSA PBP2a monoclonal antibody and centrifuged (Fig.3B), in contrast to the result for the MRSA isolate without antibody treatment 
(Fig.3A). Thus, this immunoprecipitation experiment confirmed that the 3043 Da peak was a fragment of PBP2a.

\section{DISCUSSION}

S. aureus is a major cause of hospital and communityacquired infections that can result in serious consequences (Diekema et al., 2001). Hospital infections caused by $S$. aureus include those affecting the bloodstream, lower respiratory tract, skin and soft tissues, as well as ventilator-assisted pneumonia and central

Penicillin-binding protein 2a fragment detected in this study

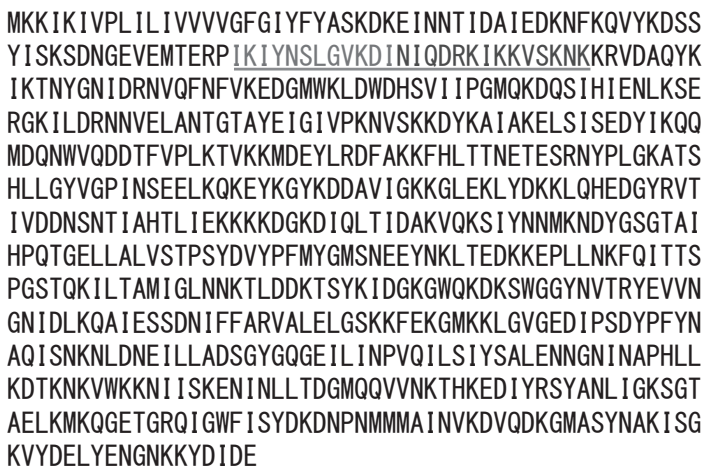

FIG. 2. N-terminal amino acid sequence of the purified fraction. Red letters show the N-terminal sequence. The underlined region has a molecular weight of $3043 \mathrm{Da}$. venous catheter-associated bacteremia. MRSA was first isolated in the UK in 1961 (Jevons., 1961) and has now become prevalent worldwide, with few countries in which MRSA is not currently present (Yoshida et al., 2003). It takes approximately 18 hours to identify S. aureus from a cultured colony using conventional methods, whereas MALDI-TOF MS enables 5-minute identification from one colony. Therefore, this method shortens the time for reporting of results to clinical sites, thereby allowing earlier selection of drugs.

Use of MALDI-TOF MS to differentiate MRSA from MSSA was first reported by Edwards-Jones et al. (Edwards-Jones et al., 2000). Intact cell mass spectra of seven reference strains of MSSA, seven clinical isolates of MRSA, and six strains of CoNs were compared in a mass range of 500-10,000 m/z. Groupspecific biomarker ions were identified in the lower mass range of 500-2000 m/z, which allowed correct differentiation of MRSA from MSSA strains (Edwards-Jones et al., 2000). Du et al. (2002) identified $74 \%$ of 76 S. aureus strains and MRSA was correctly distinguished from MSSA using cluster analysis with a dendrogram. Jackson et al. (2005) found that MSSA and MRSA in S. aureus isolates had different intensities of peaks at 3048 $\mathrm{m} / \mathrm{z}, 3086 \mathrm{~m} / \mathrm{z}$ and $3124 \mathrm{~m} / \mathrm{z}$, and Drake et al. (2011) showed that $S$. aureus isolates had strain clustering differences among community $(n=12)$ and hospitalassociated MRSA ( $n=14)$ and MSSA strain SS6 $(n=18)$, based on the intensities of peaks at $2302 \mathrm{~m} / \mathrm{z}$ and 3871 $\mathrm{m} / \mathrm{z}$. Among the 6 peaks we used to distinguish MRSA from MSSA in the present study, two $(3043 \mathrm{~m} / \mathrm{z}$ and

(A)

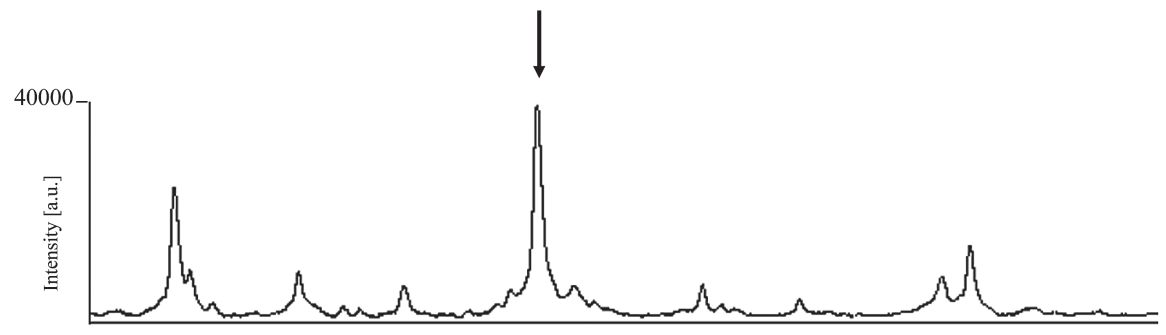

(B)

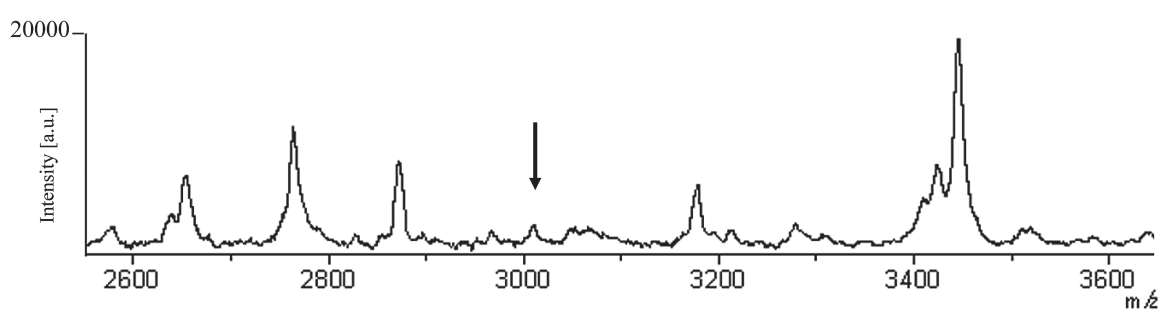

FIG. 3. Immunoprecipitation assay of peaks of interest performed to confirm the identity of the 3043 Da peak. (A) Baseline mass spectrum of a protein extract solution of a MRSA isolate treated with antibody-fixed affinity beads. (B) Mass spectrum of the supernatant after incubation of the protein extract solution of the MRSA isolate with anti-MRSA PBP2a monoclonal antibody on antibody-fixed affinity beads. The intensity of the 3043 Da peak was markedly decreased. 
$4640 \mathrm{~m} / \mathrm{z}$ ) have been reported as important peaks for distinguishing MRSA by Jackson et al. (2005) and Wolters et al. (2011).

In this study, we conducted a prospective study of routine MALDI-TOF MS analysis using a predictive model. In the blind test, $60 \mathrm{~S}$. aureus isolates (30 MSSA and 30 MRSA) were correctly classified, with identification rates of $93.3 \%$ for MSSA and $86.7 \%$ for MRSA. Among the 6 peaks in the predictive model, the 3043 $\mathrm{m} / \mathrm{z}$ peak had the highest weight and average intensity, and was identified as a fragment of PBP2a. Resistance occurs following chromosomal acquisition of novel DNA, resulting in production of PBP2a, which has a low binding affinity for methicillin (Woodford et al., 2005). PBP2a substitutes for all other PBPs, and its low affinity for all $\beta$-lactam antibiotics confers resistance to these agents, including cephalosporins (Lowy et al., 2003). PBP2a is encoded by mecA, part of the mobile genetic element in the staphylococcal chromosomal cassette mec (SCCmec) (Wolters et al., 2011). Intracellular proteins undergo repeated synthesis and degradation that cause turnover at a certain rate; however, the mechanism of PBP2a degradation by proteases is unknown.

Clinical tests such as the coagulase test only permit identification of $S$. aureus in a cultured colony. A kit for MRSA detection based on PBP2a is available, but the method is complex and time-consuming. PCR-based assays (IDI-MRSA PCR, Xpert MRSA PCR, BD GeneOhm MRSA, and Xpert MRSA/SA SSTI real-time PCR) require 1 to 4 hours from clinical sampling to obtaining results (van Hal et al., 2007; Rossney et al., 2008; Bischof et al., 2009; Wolk et al., 2009; DubouixBourandy et al., 2011). In contrast, the method proposed in this study using a predictive model enables detection from one colony in 5 minutes, and thus could be useful at clinical sites at which rapid discrimination of MRSA from MSSA is required. It should be noted that the discrimination rate was not $100 \%$, which is important at clinical sites, and further refinement of the procedures and databases are needed.

\section{ACKNOWLEDGMENTS}

This work was supported in part by a grant from the Japanese Ministry of Education, Culture, Sports, Science and Technology (No. 24590680, No.16K08976).

\section{REFERENCES}

Ben-David, D., Novikov, I., and Mermel, L. A. (2009) Are there differences in hospital cost between patients with nosocomial methicillin resistant Staphylococcus aureus bloodstream infection and those with methicillin-susceptible $S$. aureus bloodstream infection? Infect. Control Hosp. Epidemiol., 30,
453-460.

Bischof, L. J., Lapsley, L., Fontecchio, K., Jacosalem, D., Young, C., Hankerd, R., and Newton, D. W. (2009) Comparison of chromogenic media to BD GeneOhm methicillin-resistant Staphylococcus aureus (MRSA) PCR for detection of MRSA in nasal swabs. J. Clin. Microbiol. 47, 2281-2283.

Camara, J. E., and Hays, F. A. (2007) Discrimination between wild-type and ampicillin-resistant Escherichia coli by matrixassisted laser desorption/ionization time-of-flight mass spectrometry. Anal. Bioanal. Chem., 389, 1633-1638.

Diekema, D. J., Pfaller, M. A., Schmitz, F. J., Smayevsky, J., Bell, J., Jones, R. N., Beach, M., and SENTRY Partcipants Group (2001) Survey of infections due to Staphylococcus species: frequency of occurrence and antimicrobial susceptibility of isolates collected in the United States, Canada, Latin America, Europe, and the Western Pacific region for the SENTRY Antimicrobial Surveillance Program, 19971999. Clin. Infect. Dis. 32, S114-S132.

Drake, R. R., Boggs, S. R., and Drake, S. K. (2011) Pathogen identification using mass spectrometry in the clinical microbiology laboratory. J. Mass Spectrom., 46, 1223-1232.

Du, Z., Yang, R., Guo, Z., Song, Y., and Wang, J. (2002) Identification of Staphylococcus aureus and determination of its methicillin resistance by matrix-assisted laser desorption/ionization time-of-flight mass spectrometry. Anal. Chem. 74, 5487-5491.

Dubouix-Bourandy, A., de Ladoucette, A., Pietri, V., Mehdi, N., Benzaquen, D., Guinand, R., and Gandois, J. M. (2011) Direct detection of Staphylococcus osteoarticular infections by use of Xpert MRSA/SA SSTI real-time PCR. J. Clin. Microbiol. 49, 4225-4230.

Edwards-Jones, V., Claydon, M. A., Evason, D. J., Walker, J., Fox, A. J., and Gordon, D. B. (2000) Rapid discrimination between methicillin-sensitive and methicillin-resistant Staphylococcus aureus by intact cell mass spectrometry. J. Med. Microbiol., 49, 295-300.

Fan, N. J., Gao, C. F., Zhao, G., Wang, X. L., and Liu, Q. Y. (2012) Serum peptidome patterns of breast cancer based on magnetic bead separation and mass spectrometry. Diagn. Pathol. 7, 45.

Han, M., Liu, Q., Yu, J., and Zheng, S. (2010) Identification of candidate molecular markers predicting chemotherapy resistance in non-small cell lung cancer. Clin. Chem. Lab. Med., 48, 863-867.

Jackson, K. A., Edwards-Jones, V., Sutton, C. W., and Fox, A. J. (2005) Optimisation of intact cell MALDI method for fingerprinting of methicillin-resistant Staphylococcus aureus. J. Microbiol. Methods, 62, 273-284.

Jevons, M. P. (1961) Celbenin-resistant staphylococci. Br. Med. J. 1, 124-125.

Lin, H. H., Han, L. Y., Cai, C. Z., Ji, Z. L., and Chen, Y. Z. (2006) Prediction of transporter family from protein sequence by support vector machine approach. Proteins, 62, 218-231.

Liu, Y. (2004) Active learning with support vector machine applied to gene expression data for cancer classification. J. Chem. Inf. Comput. Sci., 44, 1936-1941.

Lowy, F. D. (2003) Antimicrobial resistance: the example of Staphylococcus aureus. J. Clin. Invest. 111, 1265-1273.

Niki, Y., Hanaki, H., Matsumoto, T., Yagisawa, M., Kohno, S., Aoki, N., Watanabe, A., Sato, J., Hattori, R., Terada, M., Koashi, N., Kozuki, T., Maruo, A., Morita, K., Ogasawara, K., Takahashi, Y., Watanabe, J., Takeuchi, K., Fujimura, S., Takeda, H., Ikeda, H., Sato, N., Niitsuma, K., Saito, M., Koshiba, S., Kaneko, M., Miki, M., Nakanowatari, S., Honda, Y., Chiba, J., Takahashi, H., Utagawa, M., Kondo, T., Kawana, A., Konosaki, H., Aoki, Y., Ueda, H., Sugiura, 
H., Ichioka, M., Goto, H., Kurai, D., Okazaki, M., Yoshida, K., Yoshida, T., Tanabe, Y., Kobayashi, S., Okada, M., Tsukada, H., Imai, Y., Honma, Y., Nishikawa, K., Yamamoto, T., Kawai, A., Kashiwabara, T., Takesue, Y., Wada, Y., Nakajima, K., Miyara, T., Toda, H., Mitsuno, N., Sugimura, H., Yoshioka, S., Kurokawa, M., Munekawa, Y., Nakajima, H., Kubo, S., Ohta, Y., Mikasa, K., Maeda, K., Kasahara, K., Koizumi, A., Sano, R., Yagi, S., Takaya, M., Kurokawa, Y., Kusano, N., Mihara, E., Kuwabara, M., Fujiue, Y., Ishimaru, T., Matsubara, N., Kawasaki, Y., Tokuyasu, H., Masui, K., Negayama, K., Ueda, N., Ishimaru, M., Nakanishi, Y., Fujita, M., Honda, J., Kadota, J., Hiramatsu, K., Aoki, Y., Nagasawa, Z., Suga, M., Muranaka, H., Yanagihara, K., Fujita, J., Tateyama, M., Sunakawa, K., and Totsuka, K. (2009) Nationwide surveillance of bacterial respiratory pathogens conducted by the Japanese Society of Chemotherapy in 2007: General view of the pathogens' antibacterial susceptibility. J. Infect. Chemother., 15, 156-167.

Nomura, F. (2015) Proteome-based bacterial identification using matrix-assisted laser desorption ionization-time of flight mass spectrometry (MALDI-TOF MS) : A revolutionary shift in clinical diagnostic microbiology. Biochim. Biophys. Act., 1854, 528-537.

Rossney, A. S., Herra, C. M., Brennan, G. I., Morgan, P. M., and O'Connell, B. (2008) Evaluation of the Xpert methicillin-resistant Staphylococcus aureus (MRSA) assay using the GeneXpert real-time PCR platform for rapid detection of MRSA from screening specimens. J. Clin. Microbiol., 46, 3285-3290.

Ryzhov, V., Hathout, Y., and Fenselau, C. (2000) Rapid characterization of spores of Bacillus cereus group bacteria by matrix-assisted laser desorption-ionization time-of-flight mass spectrometry. Appl. Environ. Microbiol., 66, 38283834.

Seng, P., Drancourt, M., Gouriet, F., La Scola, B., Fournier, P. E., Rolain, J. M., and Raoult, D. (2009) Ongoing revolution in bacteriology: routine identification of bacteria by matrixassisted laser desorption ionization time-of-flight mass spectrometry. Clin. Infect. Dis., 49, 543-551.

Sogawa, K., Watanabe, M., Sato, K., Segawa, S., Ishii, C., Miyabe, A., Murata, S., Saito, T., and Nomura, F. (2011) Use of the MALDI BioTyper ${ }^{T M}$ system with MALDI-TOF mass spectrometry for the rapid identification of microorganisms. Anal. Bioanal. Chem., 400, 1905-1911.

Sogawa, K., Watanabe, M., Sato, K., Segawa, S., Miyabe, A.,
Murata, S., Saito, T., and Nomura, F. (2012) Rapid identification of microorganisms by mass spectrometry: improved performance by incorporation of in-house spectral data into a commercial database. Anal. Bioanal. Chem., 403, 18111822.

van Hal, S. J., Stark, D., Lockwood, B., Marriott, D., and Harkness, J. (2007) Methicillin-resistant Staphylococcus aureus (MRSA) detection: comparison of two molecular methods (IDI-MRSA PCR assay and GenoType MRSA Direct PCR assay) with three selective MRSA agars (MRSA ID, MRSASelect, and CHROMagar MRSA) for use with infection-control swabs. J. Clin. Microbiol., 45, 24862490.

Weinstein, M. P., Towns, M. L., Quartey, S. M., Mirrett, S., Reimer, L. G., Parmigiani, G., and Reller, L. B. (1997) The clinical significance of positive blood cultures in the 1990s: a prospective comprehensive evaluation of the microbiology, epidemiology, and outcome of bacteremia and fungemia in adults. Clin. Infect. Dis., 24, 584-602.

Wisplinghoff, H., Bischoff, T., Tallent, S. M., Seifert, H., Wenzel, R. P., and Edmond, M. B. (2004) Nosocomial bloodstream infections in US hospitals: analysis of 24,179 cases from a prospective nationwide surveillance study. Clin. Infect. Dis., 39, 309-317.

Wolk, D. M., Marx, J. L., Dominguez, L., Driscoll, D., and Schifman, R. B. (2009) Comparison of MRSASelect Agar, CHROMagar Methicillin-Resistant Staphylococcus aureus (MRSA) Medium, and Xpert MRSA PCR for detection of MRSA in Nares: diagnostic accuracy for surveillance samples with various bacterial densities. J. Clin. Microbiol. 47, 3933-3936.

Wolters, M., Rohde, H., Maier, T., Belmar-Campos, C., Franke, G., Scherpe, S., Aepfelbacher, M., and Christner, M. (2011) MALDI-TOF MS fingerprinting allows for discrimination of major methicillin-resistant Staphylococcus aureus lineages. Int. J. Med. Microbiol., 301, 64-68.

Woodford, N. (2005) Biological counterstrike: antibiotic resistance mechanisms of Gram-positive cocci. Clin. Microbiol. Infect., 11, 2-21.

Yoshida, R., Kuwahara-Arai, K., Baba, T., Cui, L., Richardson, J. F., and Hiramatsu, K. (2003) Physiological and molecular analysis of a mecA-negative Staphylococcus aureus clinical strain that expresses heterogeneous methicillin resistance. J. Antimicrob. Chemother. 51, 247-255. 